\title{
PIAS1 confers DNA-binding specificity on the Msx1 homeoprotein
}

\author{
Hansol Lee, ${ }^{1,3}$ John C. Quinn, ${ }^{1}$ Kannanganattu V. Prasanth ${ }^{5}$ Victoria A. Swiss, ${ }^{1,4}$ \\ Kyriakos D. Economides, ${ }^{1}$ Marie M. Camacho, ${ }^{1}$ David L. Spector, ${ }^{5}$ and Cory Abate-Shen ${ }^{1,2,3,6}$ \\ ${ }^{1}$ Center for Advanced Biotechnology and Medicine, ${ }^{2}$ The Cancer Institute of New Jersey, ${ }^{3}$ Departments of Medicine and \\ Neuroscience, ${ }^{4}$ Graduate Program in Biochemistry, University of Medicine and Dentistry of New Jersey-Robert Wood \\ Johnson Medical School, Piscataway, New Jersey 08854, USA; ${ }^{5}$ Cold Spring Harbor Laboratory, Cold Spring Harbor, \\ New York 11724, USA
}

The mechanisms by which homeoproteins bind selectively to target genes in vivo have long remained unresolved. Here we report that PIAS1 confers DNA-binding specificity on the Msx1 homeoprotein by regulating its subnuclear localization and proximity to target genes. We demonstrate that the interaction of Msx1 with PIAS1, but not its sumoylation, is required for Msx1 to function as an inhibitor of myoblast differentiation through repression of myogenic regulatory genes, such as MyoD. We find that PIAS1 enables Msx1 to bind selectively to a key regulatory element in MyoD, the CER, in myoblast cells and to distinguish the CER from other nonregulatory TAAT-containing sequences. We show that PIAS1 is required for the appropriate localization and retention of Msx1 at the nuclear periphery in myoblast cells. Furthermore, we demonstrate that myogenic regulatory genes that are repressed by Msx1, namely $M y o D$ and $M y f 5$, are located at the nuclear periphery in myoblast cells. We propose that a key regulatory event for DNA-binding specificity by homeoproteins in vivo is their appropriate targeting to subnuclear compartments where their target genes are located, which can be achieved by cofactors such as PIAS1.

[Keywords: Homeobox genes; protein-protein interactions; myoblast differentiation; PIAS genes; DNA-binding specificity; sumoylation]

Supplemental material is available at http://www.genesdev.org.

Received November 14, 2005; revised version accepted January 31, 2006.

Homeobox genes encode an extensive family of transcriptional regulators that control a myriad of developmental processes (Manley and Levine 1985; Gehring 1987; Levine and Hoey 1988; Krumlauf 1994). Although homeoproteins display exquisite specificities in vivo, presumably reflecting their selective regulation of target genes, they display broad DNA-binding specificities in vitro (Hayashi and Scott 1990). While it is widely believed that target gene specificity in vivo is achieved through selective protein interactions (Mann and Affolter 1998), unraveling the mechanistic details has proven to be a daunting task.

The vertebrate Msx homeobox genes play key roles in regulating cellular differentiation during development. This family consists of three members, of which Msx1 and Msx2 are closely related with respect to sequence (Supplementary Fig. 1A), expression patterns, and biochemical and biological functions (Davidson 1995; Bendall and Abate-Shen 2000). Despite their expression in

${ }^{6}$ Corresponding author.

E-MAIL abate@cabm.rutgers.edu; FAX (732) 235-5789

Article and publication are at http://www.genesdev.org/cgi/doi/10.1101/ gad.1392006. tissues of unrelated embryological origin, a unifying feature of Msx expression is its restriction to progenitor populations that ultimately give rise to terminally differentiated cells (Bendall and Abate-Shen 2000). This is exemplified in the muscle lineage, where Msx 1 is transiently expressed in myoblast precursor cells but downregulated upon terminal differentiation (Bendall et al. 1999; Houzelstein et al. 1999). In accordance with its inverse correlation of expression and cellular differentiation, forced expression of Msx1 prevents precursor cells from undergoing terminal differentiation (Song et al. 1992; Woloshin et al. 1995; Bendall et al. 1999; Odelberg et al. 2000; Hu et al. 2001), while mutations of MSX1 and MSX2 are responsible for several human genetic disorders that are manifested as abnormalities of differentiation (Jabs et al. 1993; Vastardis et al. 1996; van den Boogaard et al. 2000; Wilkie et al. 2000; Jumlongras et al. 2001).

Msx homeoproteins function as regulators of differentiation through their activities as transcriptional repressors. In particular, inhibition of myoblast differentiation by Msxl reflects, in part, its ability to repress the expression of myogenic regulatory genes, such as MyoD (Woloshin et al. 1995; Bendall et al. 1999), by binding to 
a key regulatory element in the $M y o D$ promoter called the core enhancer region (CER) (Goldhamer et al. 1992; Kucharczuk et al. 1999; Lee et al. 2004). Binding of Msx1 to the CER, as well as transcriptional repression and inhibition of differentiation, requires the selective interaction of Msx 1 with a linker histone, mouse H1b (human H1e) (Lee et al. 2004). In contrast to its selective interaction with the CER in myoblast cells, Msxl can interact in vitro with many DNA sites containing a TAAT core motif (Catron et al. 1993), which is typical of other homeoproteins (Scott et al. 1989). This contrast emphasizes the important distinction between the apparent DNA-binding properties of Msxl in vitro and its actual function in myoblast cells.

In pursuit of Msx1-interacting proteins that contribute to DNA-binding specificity in a cellular context, we have now isolated PIAS1 by yeast two-hybrid screening. PIAS1 is a member of a family of at least five genes and/or spliced variants (PIAS1, PIAS3, PIASy, PIASx $\alpha$, PIAS x $\beta$ ), one of which $(P I A S \times \beta)$ was isolated as a protein partner for Msx2 (and called Miz-1, for Msx-2-interacting protein 1) (Wu et al. 1997). PIAS proteins were initially described as inhibitors of STAT signaling, hence their name protein inhibitor of activated STATs (Liu et al. 1998). Subsequent studies revealed that PIAS proteins have numerous partners, many of which are transcription factors (Schmidt and Muller 2003). The consequences of these interactions are often manifested as transcriptional repression (Schmidt and Muller 2003) and PIAS proteins have themselves been shown to interact with DNA via an $\mathrm{N}$-terminal region called the SAP domain (Fig. 1A; Sachdev et al. 2001). Further insights regarding their mechanisms of repression were provided by the discovery that PIAS proteins can function as E3 ligases for sumoylation mediated by a ring-finger domain in the C-terminal region (Fig. 1A; Kahyo et al. 2001; Sachdev et al. 2001; Kotaja et al. 2002; Schmidt and Muller 2002). We now report that Msx 1 interacts with PIAS1, which results in modification of Msx1 by sumoylation. We find that the interaction of Msx1 with PIAS1, but not its sumoylation, is required for transcriptional repression and inhibition of differentiation. PIAS1 facilitates the selective interaction of Msxl with a key regulatory element on the $M y o D$ promotor and results in the coincident localization of Msx 1 with the $M y o D$ gene, as well as the Myf5 gene, at the nuclear periphery. Thus, our findings highlight a new role for PIAS1 proteins as regulators of homeoprotein DNA-binding specificity through their ability to control the localization of homeoproteins within the nucleus and their proximity to target genes.

\section{Results}

Msx1 interacts specifically with PIAS1

Among the genes that we found to interact with Msx1 by yeast two-hybrid screening, we focused on PIAS1 because (1) we had also isolated $U b c 9$, which encodes an E2 enzyme in the sumoylation pathway (Seeler and Dejean 2003; Hay 2005); and (2) Msx2 had been previously shown to interact with another member of the PIAS family, PIASx $\beta$ (Miz-1) (Wu et al. 1997). We verified the interaction of Msxl with endogenous PIAS1, as well as with the exogenous protein, by coimmunoprecipitation assays (Fig. 1B). By analyses of truncated Msx1 proteins, we found that the C-terminal region of Msx1 was necessary for interaction with PIAS1 (Fig. 1A,C); in fact, this interaction was disrupted by deletion of the last five amino acids of Msx1 (Supplementary Fig. 2A). Conversely, analyses of truncated PIAS1 proteins in vitro revealed that an N-terminal region (amino acids 1-200), including the SAP domain, was necessary for interaction with Msx1 (Fig. 1D; Supplementary Fig. 2B).

The interaction of Msx1 with PIAS1 was highly specific since no such interaction was observed with several other homeoproteins, including its closest homolog Msx2 (Fig. 1E). This prompted us to further verify that Msx1, but not Msx2, interacted with PIAS1 (Supplementary Fig. 1B). Conversely, we found that Msx1 did not interact with PIASx $\beta$, which was shown previously to interact with Msx2 (Fig. 1F; Wu et al. 1997). Taken together, these findings demonstrate that the interaction between Msx1 and PIAS1 is highly selective, even among members of their respective protein families. To our knowledge this is the first biochemical activity that discriminates Msx1 and Msx2, which are otherwise virtually indistinguishable (Catron et al. 1996; Bendall and Abate-Shen 2000).

The reported expression patterns of Msx1 and PIAS genes, including PIAS1, are largely overlapping during mouse development (Bendall and Abate-Shen 2000; Sturm et al. 2000) and Msx1 and PIAS1 are coexpressed in undifferentiated C2C12 myoblast cells (Supplementary Fig. 3). Moreover, both Msx1 and PIAS1 are downregulated during terminal differentiation of these cells, as is evident from the appearance of myotubes and by expression of myogenin, a marker of muscle cell differentiation (Supplementary Fig. 3; Olson and Klein 1994). Therefore, Msx1 and PIAS1 are coexpressed in biologically relevant contexts.

The interaction of Msx1 with PIAS1, but not its modification by sumoylation, is required for Msx1 activity

Considering that PIAS proteins function as E3 SUMO ligases (Kahyo et al. 2001; Sachdev et al. 2001; Kotaja et al. 2002; Schmidt and Muller 2002), we next investigated whether the interaction of Msxl with PIAS1 resulted in its modification by sumoylation. We found that Msx1 was indeed sumoylated in 293T cells (Fig. 1G). This modification was stimulated by exogenous PIAS1 (Supplementary Fig. 4A), while an Msx1 protein lacking the PIAS1-interacting region [Msx1(1-271)] was not sumoylated (Fig. 1G). Detection of exogenous Myc-SUMO revealed two modified forms of Msx1 (indicated by asterisks in Fig. 1G), the upper one being predominant, as well as additional slower-mobility forms [indicated by SUMO(n) in Fig. 1G], which likely represent multimeric SUMO-modified Msx1 proteins (Fig. 1G). Accordingly, 


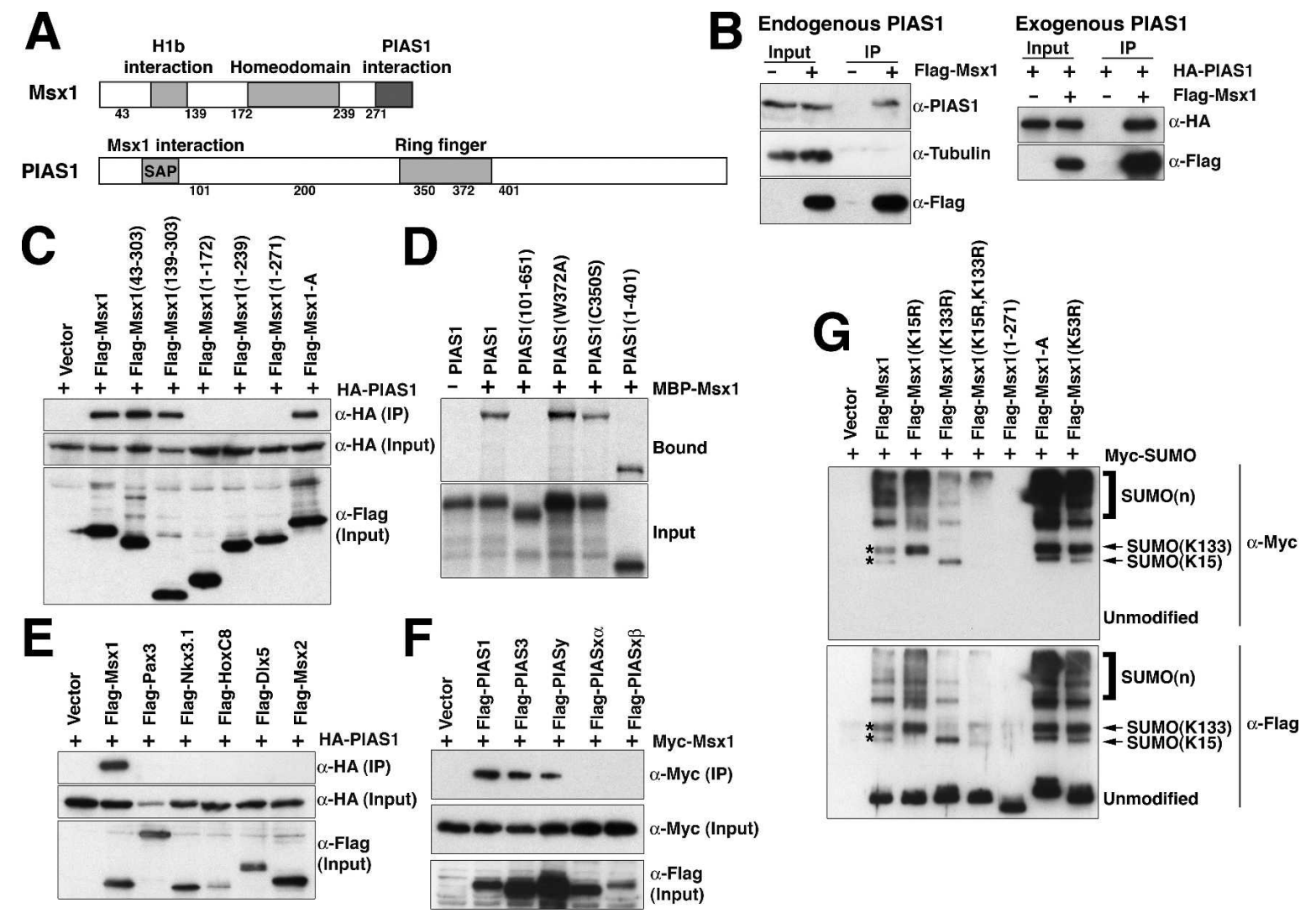

Figure 1. Msx1 interacts specifically with PIAS1. (A) Schematic diagrams of Msx1 and PIAS1. Functional domains of Msx1 (shaded boxes) include the homeodomain and the histone H1b and PIAS1 interaction regions. Functional domains of PIAS1 include the SAP domain, which interacts with DNA, and the Ring finger, which is required for E3 SUMO ligase activity. The amino acids corresponding to the truncated Msx1 and PIAS1 proteins (as shown in $C, D$; Supplementary Fig. 2) are indicated. (B) Msx1 interacts with PIAS1. 293 T cells were transfected with expression plasmids encoding Flag-Msx1 alone (Endogenous) or with HA-PIAS1 (Exogenous) followed by immunoprecipitation with $\alpha$-Flag and immunoblotting with $\alpha$-PIAS1 (top) or $\alpha$-HA (bottom) to detect the endogenous or exogenous PIAS proteins, respectively. Tubulin is shown as a control. In all experiments, lanes labeled "Input" contain $10 \%$ of the total cell extract. $(C)$ The C-terminal region of Msx1 interacts with PIAS1. 293T cells were cotransfected with HA-PIAS1 along with Flag-Msx1 or the indicated truncated Flag-tagged Msxl proteins followed by immunoprecipitation with $\alpha$-Flag and immunoblotting with $\alpha$-HA or $\alpha$-Flag. The asterisk indicates lower-mobility Msx1 proteins, which correspond in size to potential SUMO-modified forms. In all experiments, lanes labeled "Vector" contain a control plasmid. (D) The N-terminal region of PIAS1 interacts with Msx1 in vitro. PIAS1 or the indicated truncated or mutated derivatives were produced by in vitro transcription/translation and interaction assays were performed using MBP-Msx1. Bound proteins were resolved by SDS-PAGE and visualized by autoradiographic detection of the radiolabeled PIAS1 proteins. (E) Msx1, but not other homeoproteins, interacts with PIAS1. 293T cells were cotransfected with plasmids encoding HA-PIAS1 along with Flag-Msx1 or the indicated Flag-tagged homeoproteins, immunoprecipitated with $\alpha$-Flag, and probed with $\alpha$-HA or $\alpha$-Flag. $(F)$ Msx1 interacts preferentially with PIAS1. 293T cells were cotransfected with Myc-Msx1 alone or with the indicated Flag-PIAS proteins, immunoprecipitated with $\alpha$-Flag, and immunoblotted with $\alpha$-Myc or $\alpha$-Flag. $(G)$ Msxl is sumoylated at Lys 15 and Lys 133. 293T cells were transfected with Myc-SUMO along with Flag-Msx1 or the indicated Flag-Msx1 proteins. To dissociate Msx1 from other sumoylated proteins, cell lysates were denatured with 1\% SDS in the presence of $50 \mathrm{mM}$ of N-ethylmaleimide (NEM). Denatured lysates were diluted with 10 volumes of RIPA buffer followed by immunoprecipitation with $\alpha$-Flag and probed with $\alpha$-Myc or $\alpha$-Flag. A description of all proteins is provided in Supplementary Table 2.

Msx1 has two lysine residues in the $\mathrm{N}$-terminal region (K15 and K133) that are putative consensus sites for sumoylation ( $\psi \mathrm{KXE}$ ) (Supplementary Fig. 1A; Hochstrasser 2001; Seeler and Dejean 2003; Hay 2005). Substitution of these, but not other, lysine residues with arginine [Msx1(K15R); Msx1(K133R); and Msx1(K15R,K133R)] rendered Msx1 proteins that were defective for sumoylation (Fig. 1G; Supplementary Fig. 4A,B).

However, these sumoylation-defective Msx1 proteins [Msx1(K15R), Msx1(K133R), and Msx1(K15R,K133R)] were capable of interacting with PIAS1 (Fig. 2A; Supplementary Fig. 4C). Conversely, PIAS1 proteins with defective E3 SUMO ligase activity [HA-PIAS1(W372A) and HA-PIAS1(C350S) (Kahyo et al. 2001; Kotaja et al. 2002)] interacted with Msx1 (Fig. 1D). Thus, while PIAS1 sumoylates Msx1, this modification is not required for its interaction with Msxl.

The major sumoylation site (K133) is located within an $\mathrm{N}$-terminal region that is required for several key functions of Msx1, namely interaction with histone 


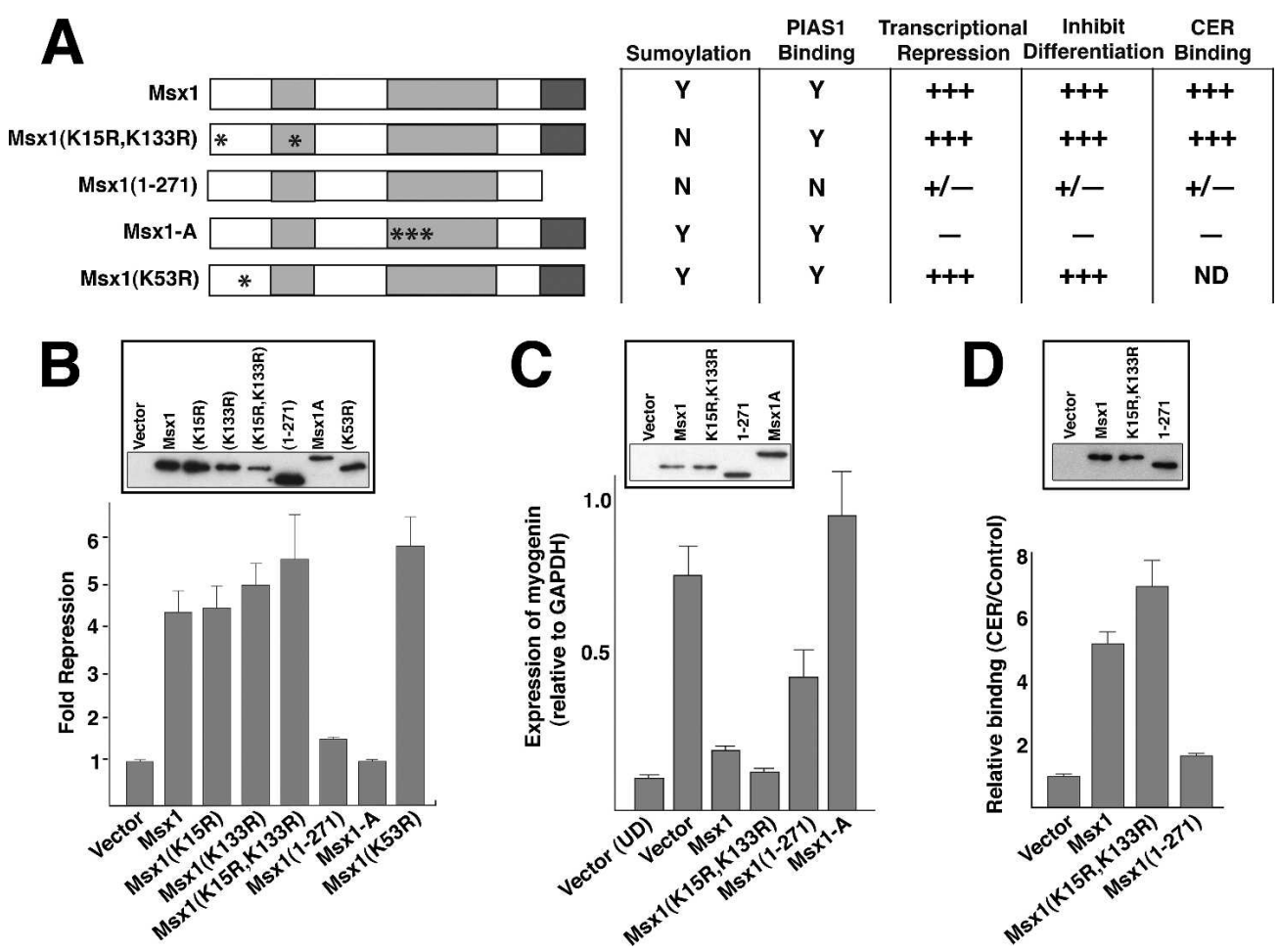

Figure 2. The interaction of Msx1 with PIAS1 is necessary for its activity. (A) Summary of the relative activities of Msx1 and truncated or mutated derivatives. The asterisks indicate the positions of amino acid substitutions. A description of the proteins is provided in Supplementary Table 2. (B) Cells were transfected with an expression plasmid expressing Msxl or the indicated Msx 1 truncated or mutated derivatives (100 ng) along with a $M y o D$ luciferase reporter plasmid (100 ng). Data are expressed as fold repression relative to the vector control. $(C)$ Cells were infected with a retrovirus expressing Msxl or the indicated Msxl derivatives and grown in conditions that promote differentiation to myotubes (Supplementary Fig. 5B). Expression of myogenin, relative to GAPDH, was determined by real-time PCR. For comparison, control cells were grown in conditions that maintain their undifferentiated state (Vector, UD). (D) Cells were infected with a retrovirus expressing Msx1 or the indicated Msx1 derivatives and grown in conditions that maintain their undifferentiated state. Binding to the CER of the endogenous MyoD gene was examined by ChIP analyses. Data were normalized to Actin as a control. Insets in $B-D$ are Western blots showing the expression levels of the exogenous Msx1 and its derivatives. Data in $B-D$ represent the averages of at least three independent experiments done in triplicate; error bars indicate standard deviation.

H1b, transcriptional repression, inhibition of differentiation, and binding to the CER regulatory element in the MyoD promoter (Supplementary Fig. 1A; Lee et al. 2004). However, we found that sumoylation of Msxl was not necessary for any of these activities (Fig. 2A-D; Supplementary Fig. 5A,B). In fact, rather than being decreased, each of these activities of the sumoyation-defective Msx1(K15R,K133R) were modestly but reproducibly increased relative to that of wild-type Msx1. In striking contrast, an Msx1 protein lacking the PIAS1 interaction region [Msx1(1-271)] was significantly attenuated in its ability to repress transcription, inhibit myoblast differentiation, and bind to the CER (Fig. 2A-D). These findings suggest that the interaction of Msxl with PIAS1, but not its sumoylation, is required for Msx1 to inhibit differentiation and repress $M y O D$ expression.

We directly investigated the requirement of PIAS for these activities of Msxl by performing knock-down experiments using short hairpin RNA interference (shRNAi) (Fig. 3). We found that depletion of endogenous PIAS1, but not PIASx $\beta$, attenuated the activities of Msx1 in transcriptional repression and inhibition of myoblast differentiation (Fig. 3A,B; Supplementary Fig. 5C; data not shown). Notably, the consequences of PIAS1 depletion were analogous to removal of the PIAS1 interaction region [Msx1(1-271)] (Fig. 2A-D). Therefore, the activities of Msx 1 in transcriptional repression and inhibition of differentiation are dependent on PIAS1.

\section{PIAS1 confers DNA-binding specificity on Msx1 in myoblast cells}

Considering that an Msx1 protein lacking the PIAS1 interaction region [Msx1(1-271)] was impaired in its ability to interact with the CER (Fig. 2D), we next asked whether PIAS1 affected DNA-binding specificity of Msx1 in myoblast cells. Inspection of sequences up to 25 $\mathrm{kb}$ upstream of the $M y o D$ promoter revealed numerous (>80) DNA sequences containing one or more TAAT motifs (Fig. 3C), which are potential Msxl-binding sites (Catron et al. 1993). While Msx1 bound to most of these TAAT sites in vitro (V.A. Swiss and C. Abate-Shen, unpubl.), it bound exclusively to the CER (fragment A) of endogenous $\mathrm{MyoD}$ in the $\mathrm{C} 2 \mathrm{C} 12$ myoblast cells (Fig. 3C; 
Figure 3. PIAS1 is required for target site recognition by Msxl in myoblast cells. $(A, B)$ PIAS1 depletion attenuates the functions of Msxl. (A) Transcriptional repression analyses were done following infection of $\mathrm{C} 2 \mathrm{C} 12$ cells with retroviruses expressing two different PIAS1 shRNAi, or a control (empty vector), and transfection with the indicated Msx1 plasmids (400 ng) and a MyoD luciferase reporter (200 ng). Data are expressed as fold repression relative to the control (vector); error bars indicate standard deviation. The $p$ values were calculated for Msx1-containing samples with the control RNAi versus PIAS1 RNAi (1) or RNAi (2). (Inset) Western blot showing knock-down of endogenous PIAS1 protein with PIAS1 shRNAi (1). Unless otherwise indicated shRNAi (1) was used for all experiments. $(B)$ Inhibition of differentiation was assessed following infection of C2C12 cells with retroviruses expressing PIAS1, PIASx $\beta$, or a control (empty vector) shRNAi along with retroviruses expressing Msx1 (or Vector) and grown in conditions that promote differentiation (Supplementary Fig. 5C). Expression of myogenin and GAPDH were determined by real-time PCR. Error bars indicate standard deviation; the $p$ value was calculated for Msx1expressing samples with the control versus PIAS1 RNAi. $(C)$ Diagram of the upstream region of the $M y o D$ gene. TAAT sequences are shown by dark boxes; the lighter box (H, DRR) has no TAAT sequences. The positions of $M y o D$ regulatory regions, CER and DRR, are indicated. Summaries of the DNA-binding activity of Msx1 in vitro (V.A. Swiss and C. Abate-Shen, unpubl.) and in vivo (Supplementary Fig. 5D) are shown. (D) PIAS1 binds selectively to the CER in C2C12 myoblast cells. ChIP assays were done in C2C12 cells infected with retroviruses expressing Flag-PIAS1, with or without Myc-Msx1. Anti-Flag was used to isolate fragments A, G, or H as indicated. Shown are representative data from two experiments done in triplicate; error bars indicate standard deviation. (E) Depletion of PIAS1 decreases the DNA-binding specificity of Msx1 in myoblast cells. ChIP assays were done in C2C12 cells following infection with retroviruses containing a control RNAi or two different PIAS1 shRNAi, or a PIASx $\beta$ shRNAi and a retrovirus expressing Flag-Msx1. Data show the average of three independent experiments done in triplicate; error bars indicate standard deviation. The $p$ values compare binding of Msx1 in cells expressing the control versus PIAS1 shRNAi (1) for sites A or G as indicated. Relative binding was determined using Actin as a control. (Inset) Western blot showing that the levels of Msxl are not altered by the PIAS1 shRNAi.

Supplementary Fig. 5D). These observations extend our previous findings (Lee et al. 2004) and demonstrate the ability of Msx1 to distinguish between a potential binding site in vitro and an authentic target site in vivo.

Importantly, we found that PIAS1 also interacts specifically and preferentially with the CER of the endogenous $M y o D$ gene in myoblast cells (Fig. 3D). Furthermore, using shRNAi to knock-down endogenous PIAS1, we found that depletion of PIAS1, but not PIASx $\beta$, resulted in decreased binding of Msxl to the CER in $\mathrm{C} 2 \mathrm{C} 12$ cells (from fourfold to 1.8 -fold, $p<0.0001$ ) (Fig. $3 \mathrm{E})$. On the contrary, depletion of PIAS1 increased binding to another TAAT-containing site (fragment G) that is not bound by Msxl in vivo (from 1.1- to 1.8-fold, $p<0.01$ ), although it did not promote binding of Msx1 to DNA sites lacking any TAAT sequences (fragment $\mathrm{H}_{\text {; }}$
Figure 3C,E). These findings suggest that PIAS1 permits Msx1 to distinguish its authentic target sites, such as the CER, from other irrelevant TAAT-containing sequences in myoblast cells.

\section{PIAS1 is required for localization of Msx1 at the nuclear periphery}

To further investigate the significance of the interaction between Msx1 and PIAS1, we examined their subnuclear localization in myoblast cells. In the absence of exogenous Msx1, exogenous PIAS1 displayed a punctate distribution within the nucleus (Fig. 4F, K), as has been reported in other cell types (Sachdev et al. 2001; Kotaja et al. 2002). However, when coexpressed with Msx1, PIAS1 exhibited a striking redistribution to the nuclear periph- 

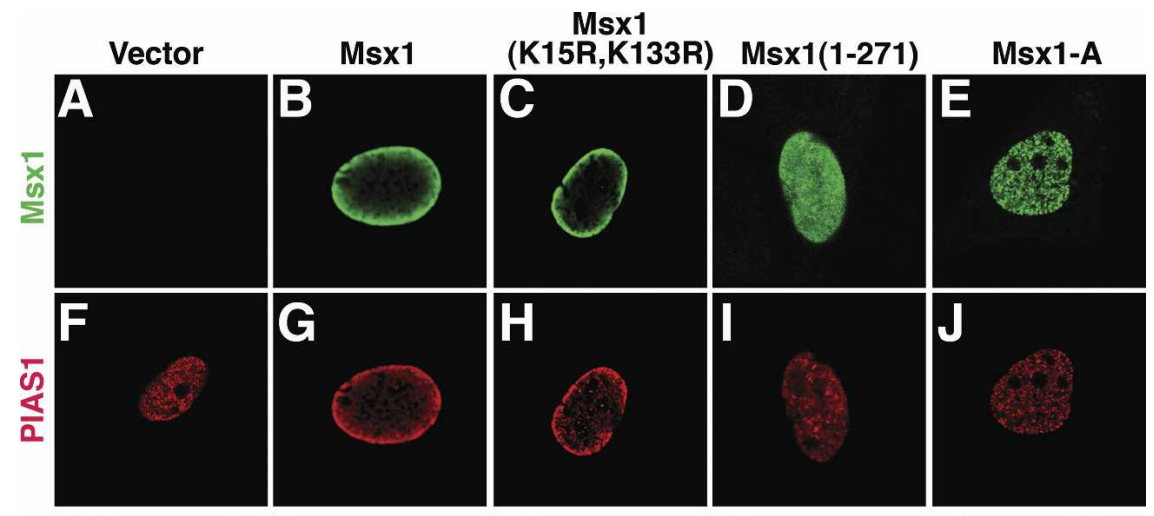
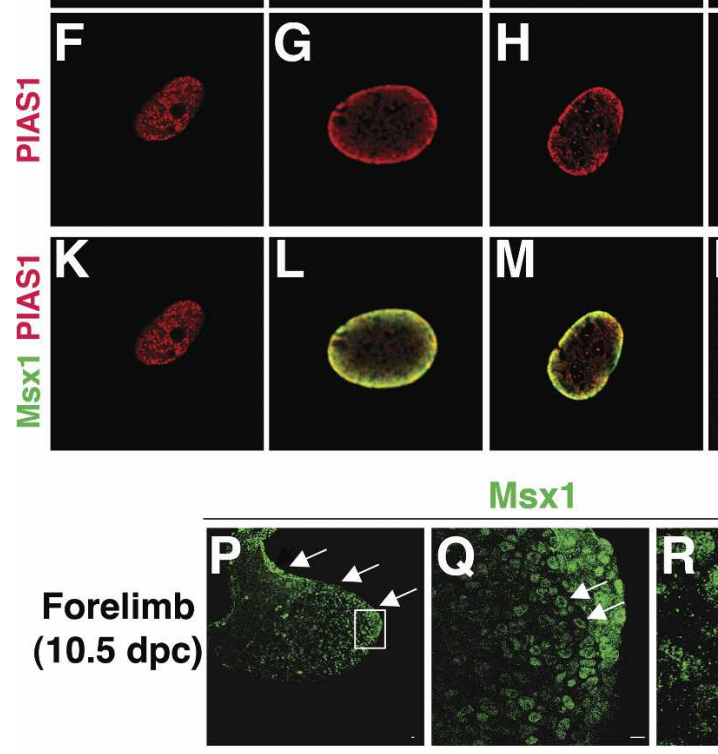

Msx1
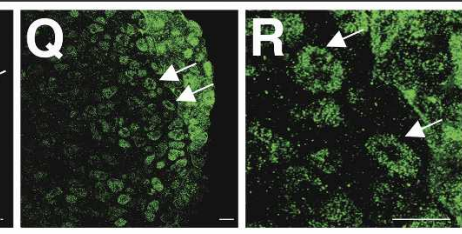

Topro3
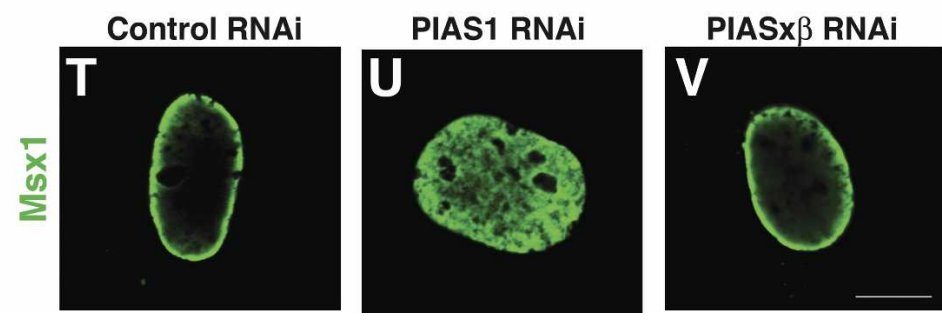

Figure 4. Colocalization of Msx1 and PIAS1 at the nuclear periphery. $(A-O)$ $\mathrm{C} 2 \mathrm{C} 12$ cells were transfected with plasmids expressing HA-PIAS1 and Flag-Msx1 or the indicated Flag-Msxl proteins (or a control vector) and visualized by immunofluorescence with $\alpha$-Flag (Msx1, green) or $\alpha$-HA (PIAS1, red). Images were collected using a Leica confocal microscope. Merged images in $\mathrm{K}-\mathrm{O}$ were generated using Adobe Photoshop. $(P-S)$ Endogenous Msx is associated with the nuclear periphery in the embryonic limb. Msx protein expression was visualized by immunofluorescence in cryosections of mouse embryos $(10.5 \mathrm{dpc}) .(P)$ Shown is the forelimb, where Msx expression is localized along the perimeter in the mesenchyme and epithelium (arrows). Higher-power views (boxed area, $P$ ) show Msx expression in the vicinity of the nuclear periphery (arrows, $Q, R)$. (S) Topro shows the staining of the nuclei. $(T-V) \mathrm{C} 2 \mathrm{C} 12$ cells expressing FlagMsx1 were infected with a retrovirus expressing a control, PIAS, or PIASx $\beta$ shRNAi. Flag-Msxl was detected by immunofluorescence with $\alpha$-Flag (Msx1, green). Note that PIAS1 depletion results in diffuse staining of Msxl throughout the nucleus rather than concentrated at the periphery. ery where it was colocalized with Msx1 (Fig. 4B,G,L). Importantly, the distribution patterns of these proteins, either individually or together, were not dependent on fixation conditions or other experimental parameters (see Materials and Methods).

We extended these studies by examining the localization of endogenous Msx1 in mouse embryos, focusing on the forelimb where both Msx1 and PIAS1 are known to be coexpressed (Bendall and Abate-Shen 2000; Sturm et al. 2000). Using a monoclonal antibody that recognizes both Msx1 and Msx2 (hence, we refer to Msx expression) (Liem et al. 1995), we observed that endogenous Msx proteins were also located at the nuclear periphery in the embryonic forelimb (Fig. 4P-S). Therefore, localization of Msxl at the nuclear periphery is not likely to be a consequence of its exogenous expression in cell culture.

Colocalization of Msx1 and PIAS1 at the nuclear periphery required their interaction, since proteins lacking their respective interaction regions were not colocalized at the periphery (Fig. 4D,I,N; Supplementary Fig. 6). In contrast, a sumoylation-defective Msx1 protein [Msx1(K15R,K133R)] and, conversely, E3 SUMO-ligase-defective PIAS1 proteins [PIAS1(W372A) and PIAS1(C350)] were colocalized with PIAS1 or Msx1, respectively, at the periphery (Fig. 4C,H,M; Supplementary Fig. $6 \mathrm{C}^{\prime}, \mathrm{D}^{\prime}, \mathrm{G}^{\prime}, \mathrm{H}^{\prime}, \mathrm{K}^{\prime}, \mathrm{L}^{\prime}$ ). Notably, Msx1-A, which is not active in transcriptional repression but interacts with PIAS1 (Figs. 1C, 2A,B; Zhang et al. 1996), was not colocalized with PIAS1 at the nuclear periphery (Fig. 4E,J,O). Taken together, these findings indicate that, regardless of its sumoylation status, Msx1 can colocalize with PIAS1 at the nuclear periphery, provided that it is able to interact with PIAS1 and function as a transcriptional repressor.

We investigated whether localization of Msxl at the nuclear periphery was dependent on endogenous PIAS1 using shRNAi knock-down. We found that depletion of PIAS1, but not PIASx $\beta$, rendered Msx1 no longer concentrated at the periphery but instead diffusely localized throughout the nucleus (Fig. 4T-V). Notably, this expression pattern was similar to that of an Msx1 protein lacking the PIAS1 interaction region [Msx1(1-271)] (Fig. 4D,I,N). Therefore, PIAS1 is necessary for localization of Msx1 to the nuclear periphery. 
Lee et al.

Figure 5. PIAS1 restricts the mobility of Msx1 within the nucleus. C2C12 cells expressing CFPMsx1 were infected with retroviruses expressing a control (empty vector), PIAS1, or PIASx $\beta$ shRNAi. (Top) Representative images from an individual cell taken prior or subsequent to photobleaching for the indicated time points; the red box shows a representative photobleached area. (Bottom) Scatterplot of the percent fluorescence recovery versus time, with $t_{1 / 2}$ indicated. Data were collected from 10-20 cells in three independent experiments; the scatterplot shows the average of these experiments. The $p$ value compares the $t_{1 / 2}$ for the PIAS1 shRNAi versus the control.

PIAS1 restricts the mobility of Msx1 within the nucleus

We extended these observations by investigating whether PIAS1 affects the kinetics of movement of Msx1 in living cells. We performed fluorescence recovery after photobleaching (FRAP) using fluorescently tagged derivatives of Msx1 [CFP-Msx1, CFP-Msx1(1-271) and CFP-Msx1(K15R,K133R)] expressed in C2C12 cells. We observed that CFP-Msx1 displayed a time for half-recovery $\left(t_{1 / 2}\right)$ in the range of 20-22 sec (Fig. 5; Supplementary Fig. 7), which is in the order of reported values for other transcriptional regulatory proteins (Carrero et al. 2003). However, following depletion of PIAS1, but not PIASx $\beta$, the mobility of CFP-Msx1 within the nucleus was significantly increased $\left(t_{1 / 2}=13 \mathrm{sec} ; p=0.01\right)$. Similarly, an Msx1 protein lacking the PIAS1 interaction region [CFPMsx1(1-271)] displayed rapid kinetics of movement within the nucleus (Supplementary Fig. 7). Although the resolution of this live-cell imaging experiment does not permit effective visualization of Msxl at the nuclear periphery, we observed that CFP-Msx1 displayed slower kinetics of movement near the periphery relative to more internal regions of the nucleus $\left(t_{1 / 2}=24 \mathrm{vs} .20 \mathrm{sec}\right.$, respectively) (Supplementary Fig. 7). These findings suggest that PIAS1 is not only required for the localization of Msxl at the nuclear periphery, it also prevents its dispersion by restricting the mobility of Msxl within the nucleus.

\section{Msx1 target genes are located in the nuclear periphery}

Considering the involvement of PIAS1 in the localization and retention of Msxl at the nuclear periphery, we next performed DNA FISH to ask where Msx1 target genes are located in myoblast cells. Strikingly, we found that $M y o D$ was localized to the nuclear periphery in $>98 \%$ (116 of 118 ) of C2C12 cells but seldom (24 of 102) in nonmyoblast cells, such as HC11 (Fig. 6A,E). This localization of $M y o D$ to the nuclear periphery was not dependent on expression of Msx1 or PIAS1 (Fig. $6 \mathrm{H}$; data not shown); however, it was dependent on the differentiation status of the cells, since $M y o D$ was not localized to the periphery in differentiated C2C12 cells (Fig. 6D).

In addition to $M y o D, M y f 5$, another myogenic regulatory gene that is repressed by Msxl (Lee et al. 2004) (Y. 

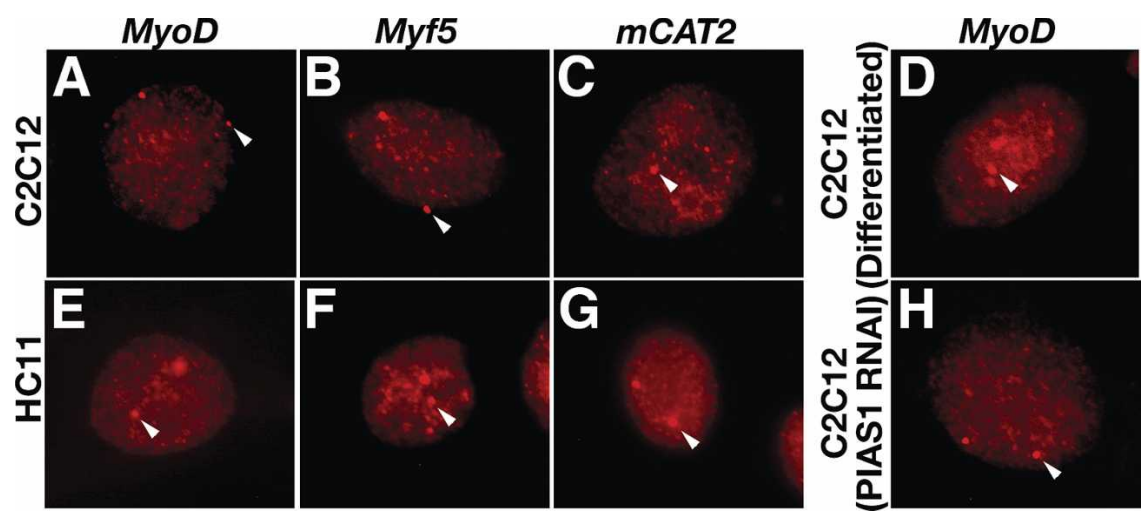

Figure 6. Target genes that are repressed by Msx1 in myoblast cells are localized to the nuclear periphery. DNA FISH analyses showing the localization (white arrowheads) of the MyoD, Myf5, or mCAT2 genes in undifferentiated myoblast cells (C2C12, A-C) and nonmyoblast cells $(\mathrm{HC} 11, E-G)$. Also shown is the localization of the $M y o D$ gene in differentiated $\mathrm{C} 2 \mathrm{C} 12$ cells $(D)$ and in undifferentiated C2C12 cells expressing a PIAS1 RNAi $(H)$.

Chen and C. Abate-Shen, in prep.), was also localized to the nuclear periphery in $>90 \%$ (58 of 63 ) of C2C12 cells but not in HC11 cells (29 of 69) (Fig. 6B,F). In contrast, mCAT2 (MacLeod 1996), a gene that is expressed in muscle cells but not repressed by Msx1 (Y. Chen and C. Abate-Shen, unpubl.), displayed no preference for localization to the nuclear periphery in either $\mathrm{C} 2 \mathrm{C} 12$ or HC11 cells (41 of 89) (Fig. 6C,G). Cumulatively, our findings suggest that PIAS1 regulates the localization Msx1, the consequences of which are to bring Msxl to the proximity of genes that are targets for its repressor function (Fig. 7).

\section{Discussion}

Understanding how homeoproteins achieve target recognition in vivo remains a critical problem in developmental biology. Indeed, bona fide target genes for homeoproteins, as well as the regulatory sequences to which they bind in vivo, remain poorly defined. To a large extent this reflects the striking contrast between the promiscuous DNA-binding specificities of homeoproteins in vitro and their selective recognition of target sites in vivo, which makes it virtually impossible to extrapolate from an in vitro binding site to a bona fide target gene. So, the challenge is to investigate the biochemical properties of homeoproteins within biologically relevant contexts.

In our analyses of the mechanisms underlying DNAbinding specificity of the Msxl homeoprotein in vivo, we have now found that PIAS1 is a critical determinant
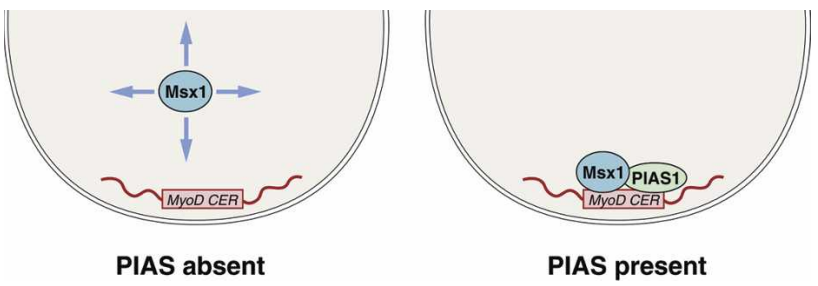

Figure 7. Model showing how PIAS1 confers DNA-binding specificity on Msx1 by regulating its proximity to target genes. In the absence of PIAS1, Msxl is free to diffuse within the nucleus and access irrelevant TAAT sites, whereas with PIAS1, Msxl is retained at the nuclear periphery where it binds specific targets, such as the CER of MyoD. of target site recognition by Msx1 in myoblast cells. Our current studies complement our previous ones in which we demonstrated that the functions of Msx1 as a transcriptional repressor and regulator of differentiation require its interaction with histone H1b (Lee et al. 2004). Notably, the interaction of Msx1 with PIAS1 occurs through a distinct domain from that required for its interaction with $\mathrm{H} 1 \mathrm{~b}$ and, unlike the interaction with $\mathrm{H} 1 \mathrm{~b}$, the interaction of Msxl with PIAS1 is not conserved with Msx2, which itself interacts with a different member of the PIAS family (Wu et al. 1997). Thus, we envision that target site recognition by Msx1 (and extrapolating more generally to other homeoproteins) represents the culmination of several distinct protein interactions, some of which are shared with Msx2 (or other homeoproteins) while others are exclusive for Msx1.

Interestingly, the E3 SUMO ligase activity of PIAS1 does not appear to be required for its ability to confer DNA-binding specificity on Msxl in vivo or for inhibition of differentiation or transcriptional repression. Although PIAS proteins sumoylate many protein partners, the consequences of this modification differ dramatically depending on the particular partner (Hochstrasser 2001; Gill 2003; Seeler and Dejean 2003; Hay 2005). In some cases, sumoylation has profound effects, as exemplified by sumoylation of Sp3, which renders it transcriptionally inactive and also affects its subnuclear localization (Ross et al. 2002; Sapetschnig et al. 2002). However, for other proteins, such as Lef1, the consequences of sumoylation are not readily apparent (Sachdev et al. 2001). In the case of Msx1, the lack of a requirement for sumoylation seems counterintuitive, since the primary sumoylation site is located within the minimal conserved region required for inhibition of differentiation and transcriptional repression (Lee et al. 2004). Indeed, rather than being less active, an Msx1 protein that is defective for sumoylation is slightly more active, which leads us to speculate that sumoylation of Msx1 may be inhibitory for these activities. One possibility is that sumoylation provides a clearance mechanism for rendering Msx1 inactive and thereby "fine-tuning" the extent and/or timing of its repressive capabilities.

While colocalization of PIAS proteins with their partners is common, colocalization to the nuclear periphery is unusual; the interaction of PIAS proteins with part- 
ners generally results in sequestration to nuclear bodies, as exemplified by the association of PIASy with the activator, Lef1 (Sachdev et al. 2001). Considering that Lef1 and Msxl have complementary biological functions in at least one biological context (Chen et al. 1996; Kratochwil et al. 1996), it is tempting to speculate that their actions are regulated, at least in part, by the ability of PIAS proteins to shuttle Lef1 and Msx1 to distinct subnuclear compartments, potentially toward or away from distinct sets of target genes.

It has long been recognized that the organization of chromosomes within the nucleus is an active process, in which chromosomes partition into distinct compartments depending on the cell type, differentiation status, and other biological parameters (Marshall et al. 1997; Vazquez et al. 2002; Harmon and Sedat 2005). Therefore, although not previously appreciated, it is perhaps not surprising that the shuttling of a homeoprotein protein to the nuclear compartment where its target genes reside plays a critical role in target recognition. Notably, the colocalization of Msx1 and PIAS1 at the nuclear periphery requires that Msxl be competent for repression, since a transcriptionally inert form of Msx1 (Msx1-A) does not colocalize with PIAS1 at the periphery. Therefore, although localization of Msx1 to the periphery requires PIAS1, Msx1 is clearly not an innocent bystander but rather an active participant in the dynamic localization of its proximity to its targets. Notably, the nuclear periphery is now recognized as a critical compartment not only for gene silencing but also for regulation of transcriptional repression (Gasser 2001; Kosak and Groudine 2004; Misteli 2004). Our findings, which implicate the nuclear periphery as the site for repression of $M y o D$ by an Msx1-PIAS1 complex in undifferentiated myoblast cells, provide further support for the idea that this nuclear compartment plays a key regulatory role in transcriptional repression.

In conclusion, we have uncovered a hitherto unappreciated function for PIAS1 as a critical determinant of homeoprotein DNA-binding specificity through its ability to regulate the localization of Msxl within the nucleus and its proximity to target genes (Fig. 7). We propose that PIAS1 retains Msx 1 at the nuclear periphery where it binds to the CER regulatory element of the $M y o D$ gene and presumably regulatory elements of other target genes, such as Myf5. Therefore, our findings support the idea that, in addition to primary sequence determinants, recognition of bona fide target sequences by Msxl requires a dynamic interplay with the nuclear architecture, which culminates in its localization to target genes. More broadly, this model can readily be extended to explain how specific target recognition occurs for other homeoproteins, regardless of whether they use PIAS proteins or analogous regulatory partners.

\section{Materials and methods}

Plasmids

Plasmids encoding Flag- or Myc-tagged Msx1, Msx1-A [Msx1(K174A,R176A,F179A)], the truncated Flag-Msx1 deriva- tives, other Flag-tagged homeoproteins, and MBP-Msx1 were described in Lee et al. (2004) and references therein. Sequences encoding Msxl derivatives with substitutions of lysine residues and those encoding the CFP-tagged Msxl proteins were generated by PCR mutagenesis. Sequences corresponding to the coding region of PIAS1 were generated by PCR from image clone \#1296138 (Research Genetics/Invitrogen) using oligonucleotides that introduced the Flag or HA epitope tags at the $\mathrm{N}$ terminus. Sequences encoding truncated PIAS1 proteins and PIAS1 proteins with substitutions of residues 372 and 350 were made by PCR. Other PIAS cDNAs were described in Liu et al. (1998). Sequences corresponding to full-length SUMO were isolated by PCR from image clones \#1547915 (SUMO2) or \#849895 (SUMO1). All data shown were obtained using SUMO2; similar results were obtained with SUMO1. All DNA sequences generated by PCR were sequence confirmed. Expression plasmids were as follows: (1) for production of MBP-tagged proteins in Escherichia coli, pMAL-c2E (New England Biolabs); (2) for transient transfection, pcDNA3 (Invitrogen); (3) for retroviral gene transfer, pLZRS-IRES-GFP (Lee et al. 2004); and (4) for retroviral delivery of shRNAi, pMSCV-HpaI-Gateway (Hemann et al. 2003). Sequences of primers used in this study are provided in Supplementary Table 1; a description of all proteins used is provided in Supplementary Table 2.

\section{Yeast two-hybrid screen}

Yeast two-hybrid screening was performed using the DupLEX-A yeast two-hybrid system (OriGene Technologies, Inc.) with the full-length Msx1 coding region fused to LexA as the bait. The library was made from mammary glands of day 12.5 pregnant mice (OriGene Technologies, Inc.), where Msx1 is known to be expressed and functionally relevant (Bendall and Abate-Shen 2000). A total of $2.7 \times 10^{6}$ transformants were screened for potential interactors. Following four independent verifications, 29 positive interactors were obtained, which corresponded to 12 independent genes. Among these were one positive clone that corresponded to PIAS1 and one that corresponded to Ubc9.

Cell culture, immunoprecipitation, and chromat in immunoprecipitation (ChIP) assays

Cell culture studies were done using human $293 \mathrm{~T}$ cells or mouse C2C12 myoblast cells. Transcriptional repression was measured by transfection of Msx1 (or other) expression plasmids along with a $M y o D$ reporter plasmid (pGL2-F3/-2.5 MyoD-promoter-luciferase) as in $\mathrm{Hu}$ et al. (2001) and Lee et al. (2004). For myoblast differentiation assays, undifferentiated $\mathrm{C} 2 \mathrm{C} 12$ cells were grown in media containing $10 \%$ serum and differentiation was induced by shifting cells to media containing $0.1 \%$ serum (Hu et al. 2001; Lee et al. 2004).

For immunoprecipitation assays, cells were lysed in RIPA buffer and proteins were immunoprecipitated with anti-Flag M2 agarose (Sigma) (Lee et al. 2004). Immunoprecipitated proteins were analyzed by immunoblotting using ECL plus Western Blotting Detection (Amersham). For the detection of endogenous PIAS1, membranes were blocked with $10 \%$ normal donkey serum (Jackson Immunological) and incubated with antiPIAS1 antibody (C-20, Santa Cruz). Other antibodies used were anti-Flag (M2, Sigma), anti-Myc (Hu et al. 2001), anti-HA (HA-7, Sigma), and anti-V5 (V5-10, Sigma).

Primers (Supplementary Table 1) were used to PCR amplify 11 fragments ( 300 base pairs each) from the upstream region of the $M y o D$ gene (as per Fig. $3 \mathrm{C}$ ) and ChIP was done as described (Lee et al. 2004). Samples were quantified by real-time PCR 
using an Mx4000 Multiplex Quantitative PCR System (Stratagene).

\section{Immunofluorescence in cells and mouse embryos}

Immunofluorescence assays were done in $\mathrm{C} 2 \mathrm{C} 12$ cells expressing Msxl and/or PIAS1 and/or the indicated PIAS shRNAi. Cells were fixed in $4 \%$ paraformaldehyde, blocked with $10 \%$ goat serum, and incubated with the following antibodies: Cy3conjugated anti-Myc (Sigma), FITC-conjugated anti-FLAG (Sigma), and anti-HA (Sigma) visualized with Texas Red (Molecular Probes). Immunofluorescence was visualized with a Leica DMIRE2/TCS SP2 inverted confocal microscope equipped with argon/krypton and helium/neon lasers using excitation wavelengths 488, 543, and $594 \mathrm{~nm}$. For Cy3 and Texas Red, the collection range of emission spectra was narrowed to eliminate the possibility of bleed-through from these two fluors. Similar results to those shown in Figure 4 and Supplementary Figure 6 were obtained in independent experiments performed in cells fixed with $10 \%$ formalin or $95 \%$ methanol $/ 5 \%$ acetic acid as well as different antibody combinations.

Immunofluorescence assays were done on cryosections of mouse embryos from post-coital day 10.5 (10.5 dpc) that had been fixed in $4 \%$ paraformaldehyde. Msx proteins were visualized using the 4G1 antibody (Developmental Studies Hybridoma Bank), which recognizes both Msx1 and Msx2 (Liem et al. 1995).

\section{FRAP assays}

C2C12 cells expressing the CFP-Msx1 with or without the indicated PIAS shRNAi were plated on $35-\mathrm{mm}$ glass-bottom microwell dishes (MatTek). FRAP was performed using a Leica confocal microscope (as above) fitted with an INC-2000 chamber/incubator (20/20 Technology, Inc.) at a constant temperature of $37^{\circ} \mathrm{C}$ and with constant levels of $\mathrm{CO}_{2}(5 \%)$. Photobleaching was performed using a 488-nm argon/krypton laser at full laser power $(100 \%$ for $0.828 \mathrm{sec})$. Three regions of interest were chosen for each cell analyzed: one in the center of the nucleus, a second near the nuclear periphery, and a third in the cytoplasm (used for calculation of background). Fluorescence recovery was monitored for a period of $300 \mathrm{sec}$ using a $63 \times$ oil objective by scanning at low laser power $(5 \%)$ at 0.828 -sec increments. Recovery curves were generated using Leica Confocal Software.

\section{DNA FISH}

C2C12 cells were pre-extracted in cytoskeletal buffer (CSK: 100 $\mathrm{mM} \mathrm{NaCl}, 300 \mathrm{mM}$ sucrose, $3 \mathrm{mM} \mathrm{MgCl}$, and $10 \mathrm{mM}$ PIPES at pH 6.8) containing $0.5 \%$ Triton-X 100 for $5 \mathrm{~min}$ on ice and fixed with $3.7 \%$ freshly prepared formaldehyde for $15 \mathrm{~min}$ at room temperature. The cells were washed in $1 \times \mathrm{PBS}(\mathrm{pH} 7.2)$ and heat denatured in $70 \%$ formamide and $2 \times$ SSC at $72^{\circ} \mathrm{C}$ for $5 \mathrm{~min}$, followed by hybridization with the nick-translated BAC probes in $2 \times$ SSC, $50 \%$ formamide, $10 \%$ dextran sulfate, yeast tRNA, and Cot- 1 DNA overnight at $37^{\circ} \mathrm{C}$ as previously described (Xing et al. 1995). BAC clones were nick-translated using spectrum red-conjugated dUTP as a substitute nucleotide according to the manufacturer's instructions (VYSIS, Inc.). The BAC clones used were as follows: MyoD, RP24-358H24; Myf5, RP23-57E8; mCAT2, RPCI-23-94B23 (all from CHORI).

\section{Acknowledgments}

The 4G1 antibody was obtained from Developmental Studies Hybridoma Bank (Iowa City, IA). We thank Drs. Eric Rubin and
Fang Liu for advice and plasmids and Dr. Yong Lin for advice on statistical analyses. We thank Drs. Marc Gartenberg, Richard Ebright, Arnold Rabson, Danny Reinberg, Aaron Shatkin, Michael Shen, and Dinah Singer for critical comments on the manuscript. This work was supported by NIH grants RO1 HD29446 to C.A.S. and GM42694 to D.L.S. K.D.E. was supported by a post-doctoral fellowship from the Department of Defense (PC040429) and V.A.S. by a predoctoral training grant in biochemistry and molecular biology (GM08360).

\section{References}

Bendall, A.J. and Abate-Shen, C. 2000. Roles for Msx and Dlx homeoproteins in vertebrate development. Gene 247: 17-31. Bendall, A.J., Ding, J., Hu, G., Shen, M.M., and Abate-Shen, C. 1999. Msx1 antagonizes the myogenic activity of Pax3 in migrating limb muscle precursors. Development 126: 49654976.

Carrero, G., McDonald, D., Crawford, E., de Vries, G., and Hendzel, M.J. 2003. Using FRAP and mathematical modeling to determine the in vivo kinetics of nuclear proteins. Methods 29: 14-28.

Catron, K.M., Iler, N., and Abate, C. 1993. Nucleotides flanking a conserved TAAT core dictate the DNA binding specificity of three murine homeodomain proteins. Mol. Cell. Biol. 13: 2354-2365.

Catron, K.M., Wang, H., Hu, G., Shen, M.M., and Abate-Shen, C. 1996. Comparison of MSX-1 and MSX-2 suggests a molecular basis for functional redundancy. Mech. Dev. 55: 185199.

Chen, Y., Bei, M., Woo, I., Satokata, I., and Maas, R. 1996. Msx1 controls inductive signaling in mammalian tooth morphogenesis. Development 122: 3035-3044.

Davidson, D. 1995. The function and evolution of Msx genes: Pointers and paradoxes. Trends Genet. 11: 405-411.

Gasser, S.M. 2001. Positions of potential: Nuclear organization and gene expression. Cell 104: 639-642.

Gehring, W.J. 1987. Homeo boxes in the study of development. Science 236: 1245-1252.

Gill, G. 2003. Post-translational modification by the small ubiquitin-related modifier SUMO has big effects on transcription factor activity. Curr. Opin. Genet. Dev. 13: 108-113.

Goldhamer, D.J., Faerman, A., Shani, M., and Emerson Jr., C.P. 1992. Regulatory elements that control the lineage-specific expression of myoD. Science 256: 538-542.

Harmon, B. and Sedat, J. 2005. Cell-by-cell dissection of gene expression and chromosomal interactions reveals consequences of nuclear reorganization. PLOS Biol. 3: e67.

Hay, R.T. 2005. SUMO: A History of Modification. Mol. Cell 18: $1-12$.

Hayashi, S. and Scott, M.P. 1990. What determines the specificity of action of Drosophila homeodomain proteins? Cell 63: $883-894$.

Hemann, M.T., Fridman, J.S., Zilfou, J.T., Hernando, E., Paddison, P.J., Cordon-Cardo, C., Hannon, G.J., and Lowe, S.W. 2003. An epi-allelic series of p53 hypomorphs created by stable RNAi produces distinct tumor phenotypes in vivo. Nat. Genet. 33: 396-400.

Hochstrasser, M. 2001. SP-RING for SUMO: New functions bloom for a ubiquitin-like protein. Cell 107: 5-8.

Houzelstein, D., Auda-Boucher, G., Cheraud, Y., Rouaud, T., Blanc, I., Tajbakhsh, S., Buckingham, M.E., Fontaine-Perus, J., and Robert, B. 1999. The homeobox gene Msx1 is expressed in a subset of somites, and in muscle progenitor cells migrating into the forelimb. Development 126: 2689-2701. 
Hu, G., Lee, H., Price, S.M., Shen, M.M., and Abate-Shen, C. 2001. Msx homeobox genes inhibit differentiation through upregulation of cyclin D1. Development 128: 2373-2384.

Jabs, E.W., Muller, U., Li, X., Ma, L., Luo, W., Haworth, I.S., Klisak, I., Sparkes, R., Warman, M.L., Mulliken, J.B., et al. 1993. A mutation in the homeodomain of the human MSX2 gene in a family affected with autosomal dominant craniosynostosis. Cell 75: 443-450.

Jumlongras, D., Bei, M., Stimson, J.M., Wang, W.F., DePalma, S.R., Seidman, C.E., Felbor, U., Maas, R., Seidman, J.G., and Olsen, B.R. 2001. A nonsense mutation in MSX1 causes Witkop syndrome. Am. J. Hum. Genet. 69: 67-74.

Kahyo, T., Nishida, T., and Yasuda, H. 2001. Involvement of PIAS1 in the sumoylation of tumor suppressor p53. Mol. Cell 8: 713-718.

Kosak, S.T. and Groudine, M. 2004. Form follows function: The genomic organization of cellular differentiation. Genes \& Dev. 18: 1371-1384.

Kotaja, N., Karvonen, U., Janne, O.A., and Palvimo, J.J. 2002. PIAS proteins modulate transcription factors by functioning as SUMO-1 ligases. Mol. Cell. Biol. 22: 5222-5234.

Kratochwil, K., Dull, M., Farinas, I., Galceran, J., and Grosschedl, R. 1996. Lef1 expression is activated by BMP-4 and regulates inductive tissue interactions in tooth and hair development. Genes \& Dev. 10: 1382-1394.

Krumlauf, R. 1994. Hox genes in vertebrate development. Cell 78: 191-201.

Kucharczuk, K.L., Love, C.M., Dougherty, N.M., and Goldhamer, D.J. 1999. Fine-scale transgenic mapping of the MyoD core enhancer: MyoD is regulated by distinct but overlapping mechanisms in myotomal and non-myotomal muscle lineages. Development 126: 1957-1965.

Lee, H., Habas, R., and Abate-Shen, C. 2004. MSX1 cooperates with histone $\mathrm{H} 1 \mathrm{~b}$ for inhibition of transcription and myogenesis. Science 304: 1675-1678.

Levine, M. and Hoey, T. 1988. Homeobox proteins as sequencespecific transcription factors. Cell 55: 537-540.

Liem Jr., K.F., Tremml, G., Roelink, H., and Jessell, T.M. 1995. Dorsal differentiation of neural plate cells induced by BMPmediated signals from epidermal ectoderm. Cell 82: 969979.

Liu, B., Liao, J., Rao, X., Kushner, S.A., Chung, C.D., Chang, D.D., and Shuai, K. 1998. Inhibition of Stat1-mediated gene activation by PIAS1. Proc. Nat1. Acad. Sci. 95: 10626-10631.

MacLeod, C.L. 1996. Regulation of cationic amino acid transporter (CAT) gene expression. Biochem. Soc. Trans. 24: 846852.

Manley, J.L. and Levine, M.S. 1985. The homeo box and mammalian development. Cell 43: 1-2.

Mann, R.S. and Affolter, M. 1998. Hox proteins meet more partners. Curr. Opin. Genet. Dev. 8: 423-429.

Marshall, W.F., Fung, J.C., and Sedat, J.W. 1997. Deconstructing the nucleus: Global architecture from local interactions. Curr. Opin. Genet. Dev. 7: 259-263.

Misteli, T. 2004. Spatial positioning; a new dimension in genome function. Cell 119: 153-156.

Odelberg, S.J., Kollhoff, A., and Keating, M.T. 2000. Dedifferentiation of mammalian myotubes induced by msx1. Cell 103: 1099-1109.

Olson, E.N. and Klein, W.H. 1994. bHLH factors in muscle development: Dead lines and commitments, what to leave in and what to leave out. Genes \& Dev. 8: 1-8.

Ross, S., Best, J.L., Zon, L.I., and Gill, G. 2002. SUMO-1 modification represses $\mathrm{Sp} 3$ transcriptional activation and modulates its subnuclear localization. Mol. Cell 10: 831-842.

Sachdev, S., Bruhn, L., Sieber, H., Pichler, A., Melchior, F., and
Grosschedl, R. 2001. PIASy, a nuclear matrix-associated SUMO E3 ligase, represses LEF1 activity by sequestration into nuclear bodies. Genes \& Dev. 15: 3088-3103.

Sapetschnig, A., Rischitor, G., Braun, H., Doll, A., Schergaut, M., Melchior, F., and Suske, G. 2002. Transcription factor Sp3 is silenced through SUMO modification by PIAS1. EMBO J. 21: 5206-5215.

Schmidt, D. and Muller, S. 2002. Members of the PIAS family act as SUMO ligases for c-Jun and p53 and repress p53 activity. Proc. Natl. Acad. Sci. 99: 2872-2877.

- 2003. PIAS/SUMO: New partners in transcriptional regulation. Cell. Mol. Life Sci. 60: 2561-2574.

Scott, M.P., Tamkun, J.W., and Hartzell III, G.W. 1989. The structure and function of the homeodomain. Biochim. Biophys. Acta 989: 25-48.

Seeler, J.S. and Dejean, A. 2003. Nuclear and unclear functions of SUMO. Nat. Rev. Mol. Cell Biol. 4: 690-699.

Song, K., Wang, Y., and Sassoon, D. 1992. Expression of Hox-7.1 in myoblasts inhibits terminal differentiation and induces cell transformation. Nature 360: 477-481.

Sturm, S., Koch, M., and White, F.A. 2000. Cloning and analysis of a murine PIAS family member, PIAS $\gamma$, in developing skin and neurons. I. Mol. Neurosci. 14: 107-121.

van den Boogaard, M.J., Dorland, M., Beemer, F.A., and van Amstel, H.K. 2000. MSX1 mutation is associated with orofacial clefting and tooth agenesis in humans. Nat. Genet. 24: 342-343.

Vastardis, H., Karimbux, N., Guthua, S.W., Seidman, J.G., and Seidman, C.E. 1996. A human MSX1 homeodomain missense mutation causes selective tooth agenesis. Nat. Genet. 13: 417-421.

Vazquez, J., Belmont, A.S., and Sedat, J.W. 2002. The dynamics of homologous chromosome pairing during male Drosophila meiosis. Curr. Biol. 12: 1473-1483.

Wilkie, A.O., Tang, Z., Elanko, N., Walsh, S., Twigg, S.R., Hurst, J.A., Wall, S.A., Chrzanowska, K.H., and Maxson Jr., R.E. 2000. Functional haploinsufficiency of the human homeobox gene MSX2 causes defects in skull ossification. Nat. Genet. 24: 387-390.

Woloshin, P., Song, K., Degnin, C., Killary, A.M., Goldhamer, D.J., Sassoon, D., and Thayer, M.J. 1995. MSX1 inhibits myoD expression in fibroblast x 10T1/2 cell hybrids. Cell 82: 611-620.

Wu, L., Wu, H., Ma, L., Sangiorgi, F., Wu, N., Bell, J.R., Lyons, G.E., and Maxson, R. 1997. Miz1, a novel zinc finger transcription factor that interacts with Msx2 and enhances its affinity for DNA. Mech. Dev. 65: 3-17.

Xing, Y., Johnson, C.V., Moen Jr., P.T., McNeil, J.A., and Lawrence, J. 1995. Nonrandom gene organization: Structural arrangements of specific pre-mRNA transcription and splicing with SC-35 domains. J. Cell Biol. 131: 1635-1647.

Zhang, H., Catron, K.M., and Abate-Shen, C. 1996. A role for the Msx-1 homeodomain in transcriptional regulation: Residues in the N-terminal arm mediate TATA binding protein interaction and transcriptional repression. Proc. Natl. Acad. Sci. 93: $1764-1769$. 


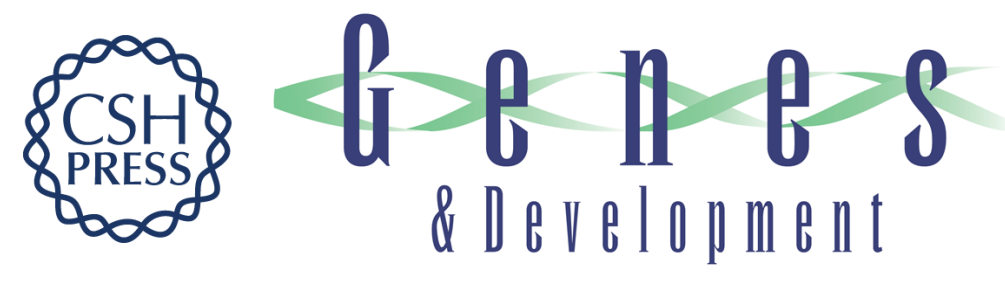

\section{PIAS1 confers DNA-binding specificity on the Msx1 homeoprotein}

Hansol Lee, John C. Quinn, Kannanganattu V. Prasanth, et al.

Genes Dev. 2006, 20:

Access the most recent version at doi:10.1101/gad.1392006

\footnotetext{
Supplemental http://genesdev.cshlp.org/content/suppl/2006/03/16/20.7.784.DC1

Material

Related Content PIAS proteins and transcriptional regulationmore than just SUMO E3 ligases?

Andrew D. Sharrocks

Genes Dev. UNKNOWN , 2006 20: 754-758

References This article cites 54 articles, 18 of which can be accessed free at: http://genesdev.cshlp.org/content/20/7/784.full.html\#ref-list-1

Articles cited in:

http://genesdev.cshlp.org/content/20/7/784.full.html\#related-urls

License

Email Alerting

Service

Receive free email alerts when new articles cite this article - sign up in the box at the top right corner of the article or click here.
}

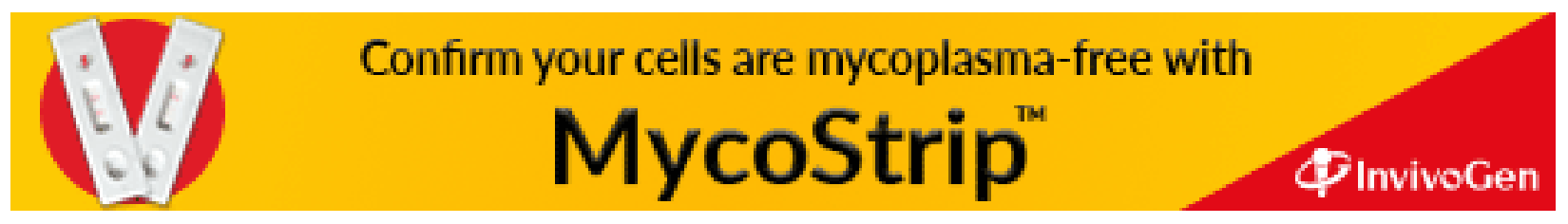

